\title{
TMEM16A regulates portal vein smooth muscle cell proliferation in portal hypertension
}

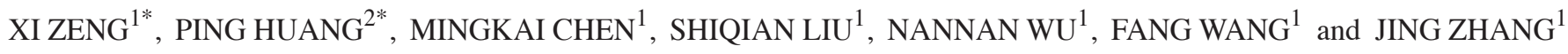 \\ ${ }^{1}$ Department of Gastroenterology, Renmin Hospital of Wuhan University, Wuhan, Hubei 430060; \\ ${ }^{2}$ Department of Gastroenterology, People's Hospital of Yichang Center, Yichang, Hubei 443003, P.R. China
}

Received March 23, 2017; Accepted September 13, 2017

DOI: $10.3892 /$ etm.2017.5466

\begin{abstract}
The aim of the present study was to elucidate the effect of transmembrane protein 16A (TMEM16A) on portal vein smooth muscle cell (PVSMC) proliferation associated with portal vein remodeling in portal hypertension (PHT). Sprague-Dawley rats were subjected to bile duct ligation to establish a rat model of liver cirrhosis and PHT. Sham-operated animals served as controls. At 8 weeks after bile duct ligation, the extent of liver fibrosis and the portal vein wall thickness were assessed using hematoxylin-eosin staining. The protein expression levels of TMEM16A, extracellular signal-regulated kinase 1 and $2($ ERK1/2) and phosphorylated ERK1/2 (p-ERK1/2) in the portal vein were detected by immunohistochemistry and western blotting. In vitro, the lentivirus vectors were constructed and transfected into PVSMCs to upregulate the expression of TMEM16A. Isolated rat primary PVSMCs were treated with a small molecule inhibitor of TMEM16A, T16A-inhA01. Cell cycle was detected by flow cytometry. The activity of TMEM16A in the portal vein isolated from bile duct ligated rats was decreased, while the expression level of p-ERK1/2 was increased. However, in vitro, upregulation of TMEM16A promoted the proliferation PVSMCs, while inhibition of TMEM16A channels inhibited the proliferation of PVSMCs. The results indicated that TMEM16A contributes to PVSMCs proliferation in vitro, but in vivo, it may be a negative regulator of cell proliferation influenced by numerous factors.
\end{abstract}

\section{Introduction}

Hepatic cirrhosis is a common response to chronic liver injury, during which the normal liver architecture is distorted by scar

Correspondence to: Professor Mingkai Chen, Department of Gastroenterology, Renmin Hospital of Wuhan University, 238 Jiefang Road, Wuhan, Hubei 430060, P.R. China

E-mail: kaimingchen@163.com

${ }^{*}$ Contributed equally

Key words: transmembrane protein 16A, portal vein smooth muscle cells, proliferation, portal vein remodeling, portal hypertension tissue and excessive extracellular matrix (ECM) is deposited (1). Portal hypertension (PHT) is a severe and frequently occurring complication of hepatic cirrhosis (2). The interaction of numerous factors in the environment forms a complex network system that affects the process of liver cirrhosis and PHT. Increased hepatic vascular resistance (HVR) to portal blood flow and increased portal collateral blood flow is the basis of the pathogenesis of PHT (3). Portal pressure increases in response to sinusoidal endothelial dysfunction (3). In addition, PHT is caused by vascular remodeling, which leads to structural and functional alteration of vessels (4). In vessels, immature vascular smooth muscle cells (VSMCs) are prone to proliferation, migration and synthesis of ECM components (5). The apoptotic decrease of portal vein smooth muscle cells (PVSMCs) in the vein wall is a cause of portal vein remodeling in PHT (6). Currently, there are no approved therapeutic options designed to reverse the progression of PHT. Therefore, it is important to search for effective methods to inhibit PHT development.

Calcium activated chloride channels ( $\mathrm{CaCCs}$ ) exist in multiple tissues and serve critical functions in fundamental physiological processes, including epithelial secretion, cardiac and neuronal excitation, regulation of smooth muscle contraction, sensory transduction, nociception and fertilization (7). In 2008, three laboratories used different approaches to demonstrate that transmembrane protein 16A (TMEM16A; also known as TAOS2 or ANO1) is a primary component of CaCCs (8-10). The coding sequence of TMEM16A is located within the 11q13 amplicon, one of the most frequently amplified chromosomal regions carrying tumor-related genes with poor prognosis, such as cyclin D1 (11). In addition to $\mathrm{Ca}^{2+}$, many factors, including calmodulin, protons, cholesterol, phosphoinositides and thermal and mechanical stimuli, could regulate TMEM16A function (7). TMEM16A dysfunction has been implicated in multiple diseases, including cancer, hypertension, gastrointestinal motility disorders and cystic fibrosis (12).

It has been confirmed that TMEM16A is present in various smooth muscle cells of ear, coronary, aortic and mesenteric arteries and the portal vein (12). The level of TMEM16A expression and its activity are significantly upregulated in hypertension and pulmonary hypertension models $(13,14)$. To the best of our knowledge, the role of TMEM16A in portal vein remodeling induced by PHT has not been studied yet. 
Numerous investigations have indicated that TMEM16A mediates tumor progression, including cell proliferation, migration and invasion (15-17). It has previously been confirmed that TMEM16A is expressed in PVSMCs (12). Factors that regulate the growth of VSMCs may contribute to the development of hypertension by promoting the thickening of vessels (18). Since TMEM16A is regarded as a regulator of cell proliferation, it is reasonable to assume that the effect of TMEM16A overexpression on PVSMC proliferation may influence PHT. Several vasoconstrictors, including urotensin II, angiotensin II (Ang II) and endothelin, have been indicated to be involved in the increased HVR in PHT (19). Consequently, an influence of TMEM16A on these and other pathogenesis pathways should also be considered and studied. Therefore, the present study aimed to determine whether TMEM16A could promote proliferation in PVSMCs to aggravate portal vein remodeling and PHT. The results indicate a functional role for TMEM16A in portal vein remodeling and PHT.

\section{Materials and methods}

Animals. All animal experimental procedures were approved by the Institutional Animal Care and Use Committee of Wuhan University (Wuhan, China) and adhered to the ethical guidelines of the International Association for the Study of Pain (20). A total of 20 male Sprague-Dawley rats, aged 6-8 weeks old and weighing 180-200 g, were purchased from the Center for Animal Experiment, Wuhan University (Wuhan, China) and maintained in specific pathogen-free conditions. The animals were housed at a constant temperature $\left(20-24^{\circ} \mathrm{C}\right)$ and humidity $(45-50 \%)$ under a 12-h light/dark cycle, and provided with food and water ad libitum. Rats were randomly assigned to two groups: The bile duct ligation (BDL) group and the control group (10 rats/group). Biliary hepatic cirrhosis was induced by ligation of the common bile duct. Briefly, rats were anesthetized with $10 \%(\mathrm{w} / \mathrm{v})$ chloral hydrate $(3.5 \mathrm{ml} / \mathrm{kg}$; Jiangsu Lianshui Pharmaceutical Co., Ltd., Lianshui, China) through intraperitoneal injection. In the BDL group, a $1.5 \mathrm{~cm}$ midline incision was made and the common bile duct was located and double ligated with $3.0 \mathrm{~mm}$ silk ligatures. Sham-operated animals in the control group received a midline incision and manipulation of the common bile duct, without ligation. All rats in each group survived the procedure. At 8 weeks following surgery, the animals were anesthetized and sacrificed by carbon dioxide euthanasia. At the time of death, the BDL was confirmed to be intact with proximal dilatation of the common bile duct. Portions of the right and left liver lobes and the portal vein were fixed in $10 \%$ buffered formalin for $24 \mathrm{~h}$ at room temperature and embedded in paraffin for histological examination.

Histological analysis. Formalin-fixed, paraffin-embedded portions of the liver lobes and portal vein were cut into $3 \mathrm{~mm}$ sections and stained with hematoxylin and eosin (H\&E) as previously described (21). Briefly, sections were deparaffinized, hydrated, stained in alum hematoxylin, differentiated with acid alcohol, washed with tap water, stained with eosin and dehydrated. Sections were then observed under a light microscope (BX51; Olympus Corporation, Tokyo, Japan), by a pathologist who was blinded to the groups. Ten high-power fields were randomly collected. The thickness of portal vein wall was quantified using Image $\mathrm{J}$ software version 1.45 (National Institutes of Health, Bethesda, MD, USA).

Immunohistochemistry was performed on sections of formalin-fixed, paraffin-embedded liver to detect expression of TMEM16A, extracellular signal-regulated kinase 1 and 2 (ERK1/2), phosphorylated ERK1/2 (p-ERK1/2). Briefly, the $3-\mu \mathrm{m}$-thick sections were prepared as described above and incubated with $3 \%$ hydrogen peroxide for $10 \mathrm{~min}$ at room temperature to block the endogenous peroxidase activity. The sections were then boiled in sodium citrate buffer $(\mathrm{pH}$ 6.0) to retrieve antigen. The sections were incubated with goat serum (Wuhan Boster Biological Technology, Ltd., Wuhan, China) for $15 \mathrm{~min}$ at room temperature. TMEM16A polyclonal antibody (cat. no. sc-69343; Santa Cruz Biotechnology, Inc., Dallas, TX, USA; 1:500 dilution), ERK1/2 monoclonal antibody (cat. no. $4695 ; 1: 400$ dilution) and p-ERK1/2 monoclonal antibody (cat. no. 4370; 1:400 dilution) (both, Cell Signaling Technology, Inc., Danvers, MA, USA) were added and incubated overnight at $4^{\circ} \mathrm{C}$. The following day, the sections were rinsed in PBS three times and incubated with horseradish-peroxidase conjugated secondary antibody (cat. no. HAF008; 1:1,000 dilution; R\&D Systems, Inc., Minneapolis, MN, USA) for $15 \mathrm{~min}$ at room temperature. A 3,3'-diaminobenzidine color developing substrate was added and the sections were examined microscopically for color development for 5-10 min, mounted and visualized under a light microscope (BX51; Olympus Corporation).

Single PVSMC isolation and culture. Primary single PVSMCs were isolated as previously described $(22,23)$. Briefly, the portal vein was dissected under sterile conditions. The connective tissues surrounding the outer membrane and endothelial cells of tunica intima were carefully stripped off under a dissecting microscope. The smooth muscle layer of the tunica media was separated from the tunica adventitia with a blunt dissection technique and then cut into small fragments $\left(1-2 \mathrm{~mm}^{3}\right)$, which were placed in $25 \mathrm{~cm}^{3}$ culture plates ( 20/plate). Dulbecco's modified Eagle's medium (DMEM) supplemented with $10 \%$ fetal bovine serum and $1 \mathrm{X}$ strength Antibiotic/Antimycotic (all Gibco; Thermo Fisher Scientific, Inc., Waltham, MA, USA) was carefully added to the culture plates so as not to disturb adhered explants. Culture plates were placed in a $37^{\circ} \mathrm{C}$ incubator $\left(5 \% \mathrm{CO}_{2}\right)$. Cells started growing from explants within 1 week and became confluent in 4 weeks. Cells were fixed for $10 \mathrm{~min}$ in glacial acetate at room temperature and immersed in $0.3 \% \mathrm{H}_{2} \mathrm{O}_{2}$ for $30 \mathrm{~min}$ to quench endogenous peroxidase activity at room temperature. They were then incubated with primary antibodies directed against smooth muscle $\alpha$-actin (cat. no. SAB2500963; 1:1,000 dilution; Sigma-Aldrich; Merck KGaA, Darmstadt, Germany) at room temperature for $1 \mathrm{~h}$ and secondary antibodies conjugated to FITC (cat. no. SAB3700002; 1:1,000 dilution; Sigma-Aldrich; Merck KGaA) for $30 \mathrm{~min}$ at room temperature. Morphometry was performed from three random fields of each slide using the Image Pro Plus software version 6.0 (Media Cybernetics Inc., Rockville, MD, USA). An Olympus-BX53 microscope (Olympus Corporation, Tokyo, Japan) was used at a magnification of x200 to observe the slides and calculate the number of smooth muscle $\alpha$-actin positive PVSMCs. 
Lentivirus infection and plasmid transfection. Recombinant pEGFP-TMEM16A and pEGFP-N1 GV358 lentivirus plasmids were packaged using Lenti-Easy Packaging mix (all Shanghai GeneChem Co., Ltd, Shanghai, China) and the virus titer was determined using methods of fluorescence enumeration, as previously described (24). Briefly, PVSMCs were plated on a 6-well plate with DMEM supplemented with $10 \%$ fetal bovine serum in a $37^{\circ} \mathrm{C}$ incubator with $5 \% \mathrm{CO}_{2}$ at a density of $1-1.5 \times 10^{5} / \mathrm{ml}$. After $24 \mathrm{~h}$, pEGFP-TMEM16A or pEGFP-N1 plasmids were transfected into the cells with Lipofectamine ${ }^{\mathrm{TM}}$ 2000 reagent (Invitrogen; Thermo Fisher Scientific, Inc.) in OPTI-MEM I reduced serum medium (Gibco; Thermo Fisher Scientific, Inc.), according to the manufacturer's protocol. After $6 \mathrm{~h}$ the cells were rinsed with PBS and switched to $5 \%$ fetal bovine serum-containing DMEM. The expression of green fluorescence protein was observed under an inverted fluorescent microscope (Olympus-IX71; Olympus Corporation) 3 days later and the transfection efficiency was calculated. The average rate of transfection=positive cells number/total number of cells $\times 100 \%$ (one visual field of the microscope) (25).

Stock solutions $(5 \mathrm{mM})$ of the TMEM16A inhibitor, T16Ainh-A01 (cat. no. SML0493; Sigma-Aldrich; Merck $\mathrm{KGaA}$ ) were made in dimethyl sulfoxide (DMSO; cat. no. D-2650-5; Sigma-Aldrich) and stored at $-20^{\circ} \mathrm{C}$. The compound was freshly diluted on the day of the experiment to a final concentration of $10 \mu \mathrm{M}$. DMSO was used at a final concentration of 1:500 for the vehicle control. Cells were treated with $10 \mu \mathrm{M}$ T16Ainh-A01 at $37^{\circ} \mathrm{C}$ in $5 \% \mathrm{CO}_{2}$ for $6 \mathrm{~h}$.

Flow cytometry. Following treatment with plasmid transfection or the TMEM16A inhibitor, T16Ainh-A01 the cells were detached from the 6-well plates with $0.2 \%$ trypsin, harvested, washed twice with PBS and centrifuged twice at $300 \mathrm{x} g$ for 5 min at $4^{\circ} \mathrm{C}$. Then, the supernatant was discarded and the pellet was resuspended in $400 \mu \mathrm{l}$ Annexin $\mathrm{V}$ binding buffer (Beyotime Institute of Biotechnology, Haimen, China) at $20^{\circ} \mathrm{C}$ for $\geq 12 \mathrm{~h}$. Cells were subsequently treated in PBS with RNase A for $30 \mathrm{~min}$ at room temperature and stained with propidium iodide. Flow cytometric analysis was performed using EPICS XL-MCL ${ }^{\mathrm{TM}}$ software (Beckman Coulter, Inc., Brea, CA, USA) and a FACScan Flow Cytometer (BD Biosciences, Franklin Lakes, NJ, USA) was used to determine the DNA contents. A concentration of $1-5 \times 10^{6} / \mathrm{ml}$ cells were analyzed for each sample, and the experiment was repeated $\geq 3$ times. The $S$-phase cell ratio and proliferation index were calculated at the same time based on the following equation: $S$-phase cell ratio $=S /\left(G_{0} / G_{1}+S+G_{2} / M\right)$; proliferation index $=\left(S+G_{2} / M\right) /\left(G_{0} / G_{1}+S+G_{2} / M\right)(26)$.

Western blotting. Western blotting analysis was performed as previously described (27). In brief, Portal vein samples were lysed in RIPA lysis buffer $(50 \mathrm{mmol} / \mathrm{l}$ Tris-HCl, $\mathrm{pH} 7.4$, $150 \mathrm{mmol} / \mathrm{l} \mathrm{NaCl}, 10 \mathrm{mmol} / \mathrm{l}$ phenylmethylsulfonyl fluoride, $1 \mathrm{mmol} / 1$ EDTA, $0.1 \%$ SDS, $1 \%$ Triton X-100 and 1\% sodium deoxycholate) for 20-30 min on ice. Protein concentrations were determined using the Lowry protein assay. Samples were then boiled in loading buffer and separated by $10 \%$ SDS-PAGE, $20 \mu \mathrm{g}$ of protein was loaded per lane. After electrophoresis, protein was transferred onto a nitrocellulose membrane, which was incubated with blocking solution [10\% non-fat dry milk in TBS containing 0.05\% Tween-20 (TBST)] for $2 \mathrm{~h}$. Membranes were immunoblotted with primary antibodies, including TMEM16A (1:1,000 dilution), ERK 1/2, p-ERK 1/2 (both 1:2,000 dilution) and $\beta$-actin (cat. no, ab8229; 1:10,000 dilution; Abcam, Cambridge, UK) overnight at $4^{\circ} \mathrm{C}$. After washing with TBST, the membranes were incubated with anti-rabbit immunoglobulin conjugated to horseradish peroxidase secondary antibody (cat. no. sc-362261; 1:1,000 dilution; Santa Cruz Biotechnology, Inc.) for $2 \mathrm{~h}$ at room temperature. The membranes were developed using an enhanced chemiluminescence western blotting kit (EMD Millipore, Billerica, MA, USA), then exposed to X-ray film. The bands of interest were quantified by Image Pro Plus version 6.0 analysis software.

Statistical analysis. Data are presented as the mean \pm standard deviation. SPSS 19.0 software (IBM Corp., Armonk, NY, USA) was used for data analysis. Statistical analysis was performed using Student's t-test for the comparison of two groups, or one-way analysis of variance followed by a Dunnett's test for multiple comparisons. $\mathrm{P}<0.05$ was considered to indicate a statistically significant difference.

\section{Results}

Establishment of the BDL model. First, it was verified that biliary hepatic cirrhosis and portal vein remodeling rats by BDL had been established successfully (Fig. 1A). H\&E staining of liver pathological sections indicated that lymphocyte infiltration and pseudolobuli formation were obvious in the liver of the BDL group. Normal structures were observed in the sham-operated group. The portal vein remodeling in PHT was characterized by thickening of the vein. The thickness of portal vein was significantly increased in the BDL group compared with the sham-operated group $(\mathrm{P}<0.05$; Fig. 1A).

Immunohistochemistry and western blot analysis of TMEM16A and p-ERK1/2. Immunohistochemistry results indicated that the TMEM16A protein expression level was decreased in BDL rats compared with the control (Fig. 1B). Western blot analysis indicated that the protein expression level of TMEM16A was significantly decreased in BDL rats ( $\mathrm{P}<0.05$; Fig. 2). p-ERK1/2 expression was indicated to be increased in BDL rats in the immunohistochemistry results (Fig. 1B) and significantly increased in the results of western blot analysis $(\mathrm{P}<0.05$; Fig. 2$)$. These results suggested that TMEM16A may have a negative association with PVSMC proliferation and portal vein remodeling.

PVSMC isolation and transfection. PVSMCs were successfully isolated and cultured. Then, pEGFP-TMEM16A and pEGFP-N1 plasmids were used to transfect PVSMCs (Fig. 3A and $\mathrm{B}$ ). The expression of TMEM16A was significantly upregulated in the pEGFP-TMEM16A transfection plasmids group compared with the control and pEGFP-N1 plasmids groups (Fig. 3C and D).

Flow cytometry. The expression of TMEM16A was upregulated by pEGFP-TMEM16A transfection plasmids as described 
A

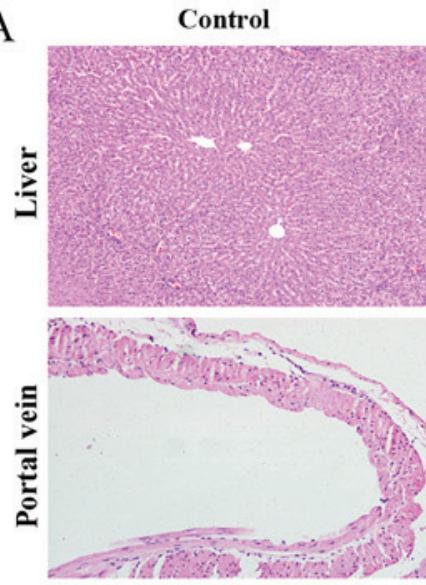

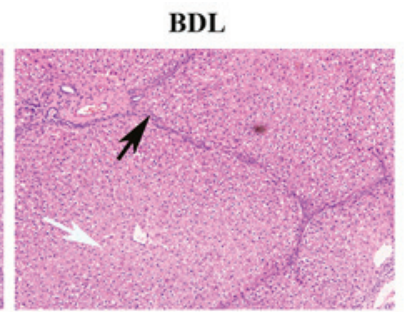

B
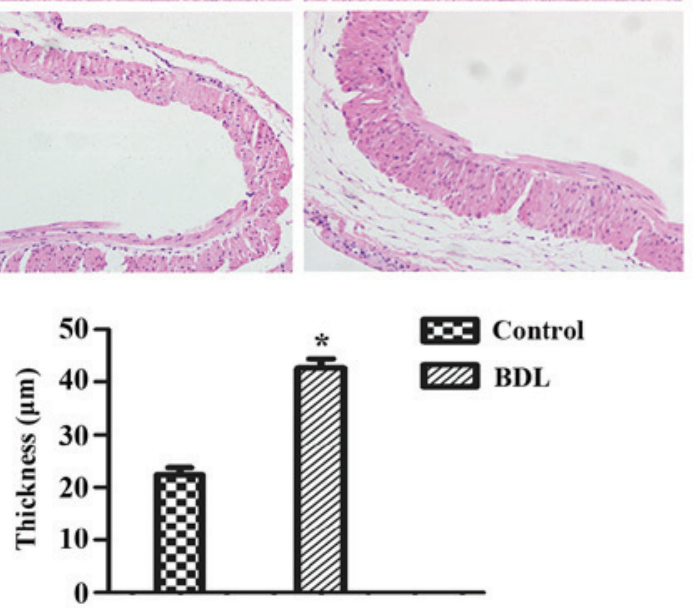
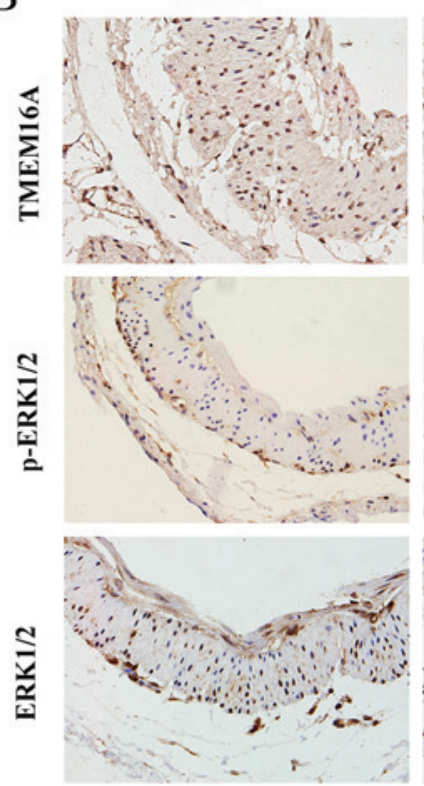

BDL

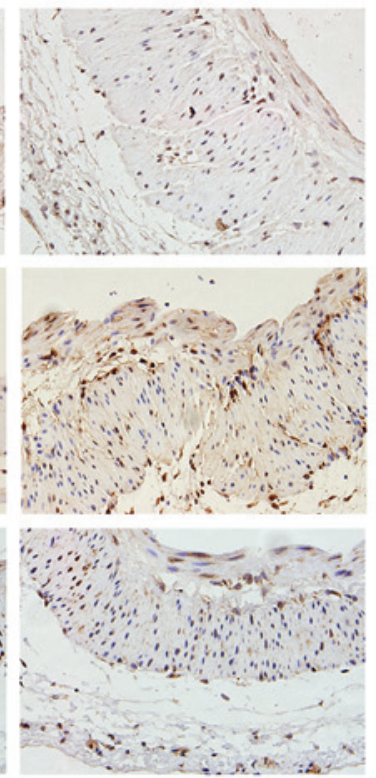

Figure 1. Pathological alterations and protein expression levels in the rat cirrhotic liver at 8 weeks after BDL. (A) The extent of liver fibrosis (magnification, $\mathrm{x} 200$ ) and the wall thickness of the portal vein (magnification, $\mathrm{x} 400$ ) were assessed by hematoxylin and eosin staining. Data are presented as the mean \pm standard deviation $(n=10)$. Compared with the normal structure of liver, severe degeneration associated with necrosis were observed in model group (white arrow), accompanied by inflammatory infiltration around the portal area, a wide range of hyperplasia in connective tissues and destruction in lobular structure (black arrow). Compared with the control group, the thickness of the portal vein in the model group was increased $(21.75 \pm 5.56 \mu \mathrm{m} v \mathrm{v}$. $43.27 \pm 9.62 \mu \mathrm{m})$. ${ }^{*} \mathrm{P}<0.05 \mathrm{vs}$. control group. (B) Protein expression levels of TMEM16A, p-ERK1/2, ERK1/2 in the portal vein were visualized by immunohistochemistry. BDL, bile duct ligation; TMEM16A, transmembrane protein 16A; ERK1/2, extracellular signal-related kinase 1 and 2; p-ERK1/2, phosphorylated ERK1/2.
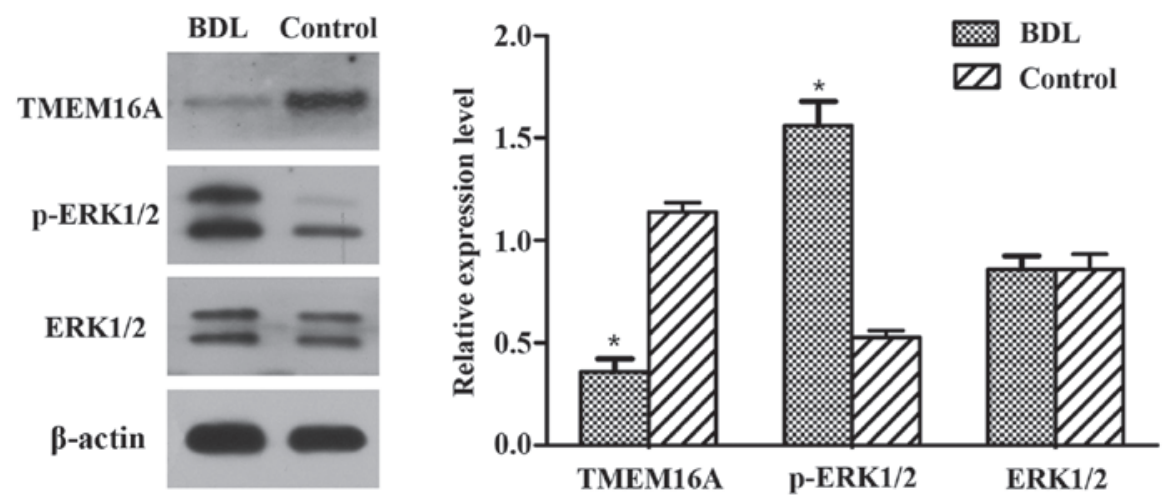

Figure 2. Protein expression levels of TMEM16A, ERK1/2 and p-ERK1/2 in BDL rats. Protein expression was detected by western blotting of portal vein sections isolated from BDL and control rats. Protein expression levels were normalized against $\beta$-actin. Experiments were performed in triplicate. Data are presented as the mean \pm standard deviation. "P<0.05 vs. control. BDL, bile duct ligation; TMEM16A, transmembrane protein 16A; ERK1/2, extracellular signal-related kinase 1 and 2; p-ERK1/2, phosphorylated ERK1/2.

above and CaCCs were inhibited by T16Ainh-A01 $(10 \mu \mathrm{M})$ as described previously (28). Compared with the negative control pEGFP-N1 plasmid group, the $\mathrm{S}$ rate $(34.44 \pm 3.72 \mathrm{vs}$. $15.56 \pm 2.36 ; \mathrm{P}<0.05)$ and proliferation index $(45.06 \pm 0.09$ vs. $20.40 \pm 0.02$; $\mathrm{P}<0.001)$ of pEGFP-TMEM16A plasmid-transfected cells were significantly increased (Fig. 4). The $S$ rate $(4.51 \pm 0.45$ vs. $16.72 \pm 0.21)$ and proliferation index $(8.59 \pm 0.02$ vs. $22.8 \pm 0.01)$ of T16Ainh-A01 treated cells were significantly reduced compared with the control group $(\mathrm{P}<0.01$; Fig. 4$)$. It was identified that overexpression of TMEM16A facilitated PVSMC proliferation, while inhibition of TMEM16A reduced
PVSMC proliferation. TMEM16A contributed to PVSMC proliferation in vitro, but in vivo, it may be a negative regulator of cell proliferation. The contradictory results in vivo and in vitro require further research.

\section{Discussion}

The primary focus of research into TMEM16A has been tumorigenesis and cancer progression (16). The fundamental role of TMEM16A in the generation of slow waves in interstitial cells of Cajal has also been demonstrated (29). Furthermore, 


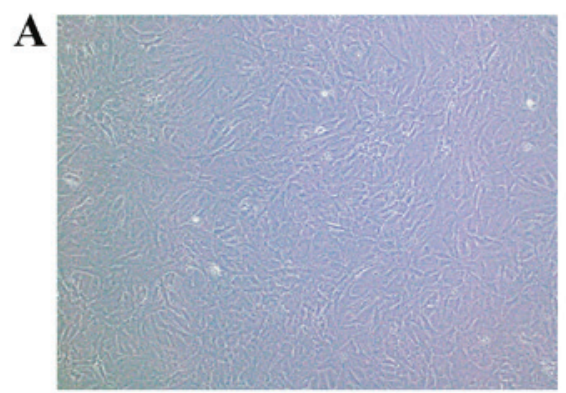

B

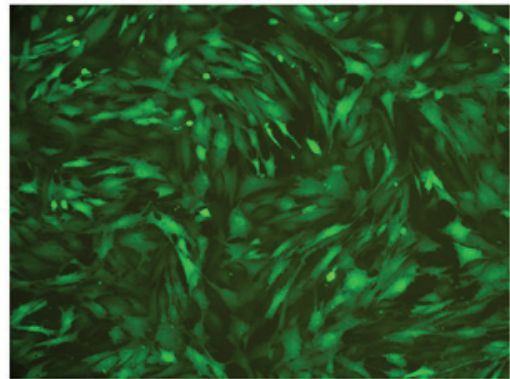

C

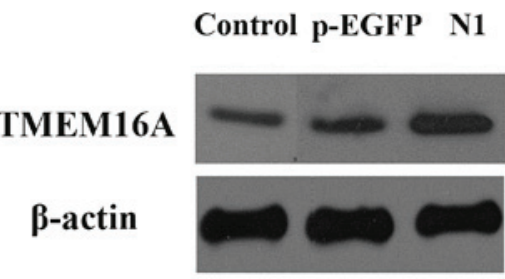

relative expression level of TMEM16A

D

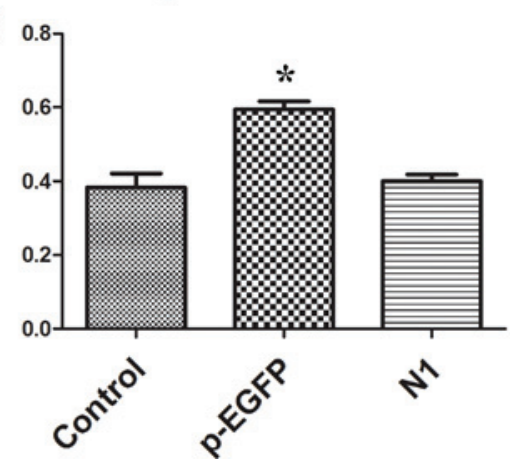

Figure 3. PVSMCs transfected with pEGFP-TMEM16A plasmid at multiplicity of infection (multiplicity of infection=100) were observed under (A) a light microscope and (B) a fluorescence microscope. Magnification, x40. (C) Protein expression levels of TMEM16A in the control, pEGFP-TMEM16A transfection plasmid and pEGFP-N1 plasmid groups. (D) Quantification of the results from part C. " $\mathrm{P}<0.05$ vs. the control. PVSMC, portal vein smooth muscle cell; TMEM16A, transmembrane protein 16A; pEGFP, pEGFP-TMEM16A plasmid group; N1, pEGFP-N1 plasmid group.
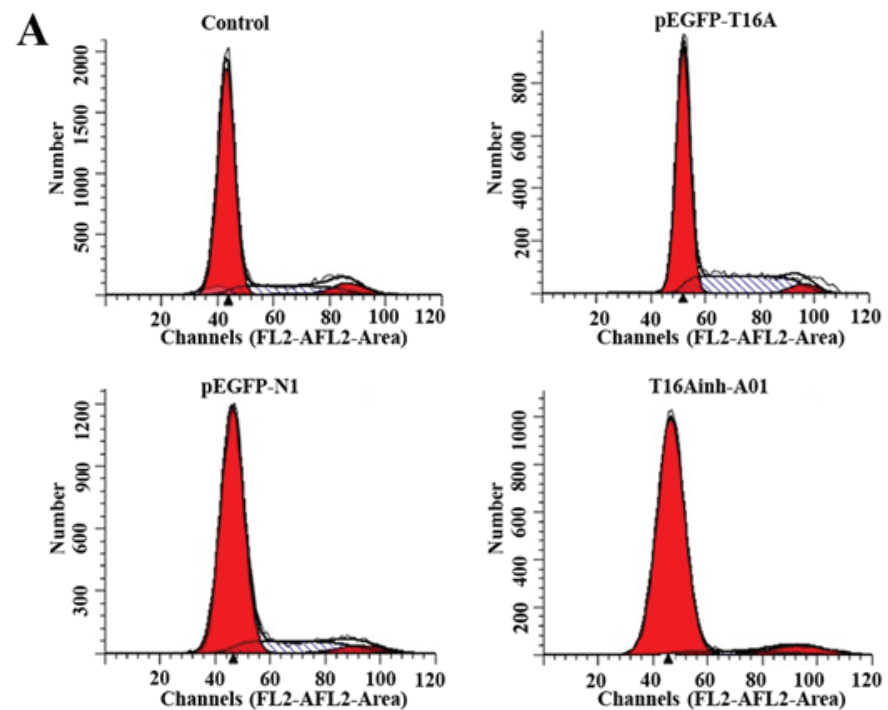

\begin{tabular}{|l|l|l|}
\hline Groups & S-phase cell ratio (\%) & Proliferation index (\%) \\
\hline Control & $16.72 \pm 0.21$ & $22.8 \pm 0.01$ \\
\hline pEGFP-T16A & $34.44 \pm 3.72^{*}$ & $45.06 \pm 0.09^{* * *}$ \\
\hline pEGFP-N1 & $15.56 \pm 2.36$ & $20.40 \pm 0.02$ \\
\hline T16Ainh-A01 & $4.51 \pm 0.45^{\Delta \Delta}$ & $8.59 \pm 0.02$ \\
\hline
\end{tabular}

Figure 4. (A) Cell cycle was detected by flow cytometry analysis. (B) The S-phase cell ratio and proliferation index were calculated at the same time based on the following equation: S-phase cell ratio $=\mathrm{S} /(\mathrm{G} 0 / \mathrm{G} 1+\mathrm{S}+\mathrm{G} 2 / \mathrm{M})$; proliferation index $=(\mathrm{S}+\mathrm{G} 2 / \mathrm{M}) /(\mathrm{G} 0 / \mathrm{G} 1+\mathrm{S}+\mathrm{G} 2 / \mathrm{M})$. Data are presented as the mean \pm stan dard deviation. ${ }^{*} \mathrm{P}<0.05 ;{ }^{* * *} \mathrm{P}<0.001$ vs. pEGFP-N1; ${ }^{\Delta \Delta} \mathrm{P}<0.01$ vs. control group.

TMEM16A is expressed in the renal collecting duct principal cells and may be involved in the physiological regulation of $\mathrm{NaCl}$ transport in vivo (30). TMEM16A can affect $\mathrm{Ca}^{2+}$ entry and VSMC contraction by regulating membrane potentials by depolarization (31). However, the association between TMEM16A and PVSMCs in PHT remains unclear.

To the best of our knowledge, the present study is the first to indicate that TMEM16A is attenuated in the PVSMCs of a widely used BDL rat model. This suggests that TMEM16A may be negatively associated with PVSMC proliferation and portal vein remodeling in PHT. This is consistent with a previous study, which reported that downregulation of TMEM16A is a major contributing factor in the remodeling of the wall of cerebral arteries in a two-kidney, two clip hypertensive rat model (32). However, in the present in vitro results, it was observed that upregulation of TMEM16A promoted PVSMC proliferation, 
while inhibition of TMEM16A reduced PVSMC proliferation. These results seem to contradict the in vivo findings.

Experimental and human studies have consistently indicated that Ang II is involved in the development of cardiac hypertrophy and pulmonary hypertension (33-36). Ang II contributes to the proliferation of hepatic stellate cells and the progression of liver fibrosis (37). Decreased vasodilatory substances, including nitric oxide and/or excessive production of vasoconstrictors, including endothelin and Ang II, was reported to increase HVR (3). Therefore, the renin Ang II system is involved in the regulation of intrahepatic vascular resistance in cirrhosis.

Wang et al (32) reported that Ang II suppresses TMEM16A expression. The study also identified that knockdown of TMEM16A facilitates and overexpression of TMEM16A inhibits Ang II-induced cell cycle transition and cell proliferation. Ang II has been reported to increase proliferation in VSMCs (38) and inhibit TMEM16A expression via Krüppel-like factor 5 in cultured VSMCs (39). A previous report that the expression of Ang II is increased in the portal vein and splenic vein in patients with liver cirrhosis (40) suggests that the expression of TMEM16A in the portal vein may be suppressed by increased Ang II in BDL rats.

Recent studies have revealed that TMEM16A promotes cancer cell proliferation and tumor growth through the mitogen-activated protein kinase (MAPK)-ERK signal pathway $(16,41)$. The present results indicated that the expression of p-ERK1/2 was increased while TMEM16A was decreased, which is in opposition to the aforementioned TMEM16A-MAPK-ERK signal pathway. An alternative possibility is that the positive effect of Ang II on p-ERK1/2 obscured the negative effect of downregulation of TMEM16A. This is supported by previous evidence that Ang II induces a substantial and rapid increase in p-ERK1/2 activity (42). In BDL rats, Ang II may serve a key function in controlling the expression of TMEM16A and the MAPK-ERK signal pathway. However, the effect of Ang II may be context-dependent. One group reported that Ang II significantly enhances TMEM16A expression in VSMCs via the Ang II type 1 receptor-phosphoinositide 3-kinase-Akt pathway (15). Nonetheless, further experiments are required to confirm whether Ang II is the negative regulator of TMEM16A expression in portal vein reconstruction.

A future focus for research will be to examine TMEM16A expression levels in the portal vein of PHT patients to provide insight into the clinical significance of TMEM16A in PHT pathogenesis. The present study identified that a $\mathrm{CaCC}$ channel, TMEM16A, is downregulated in the portal vein of PHT rats in vivo. However, TMEM16A was also observed to promote PVSMC proliferation in vitro. The regulatory peptide Ang II may be involved in regulating these opposite situations. This study indicates a potential mechanism of PHT and provides a basis for further research into the function of TMEM16A in PVSMC proliferation.

\section{References}

1. Intengan HD and Schiffrin EL: Vascular remodeling in hypertension: Roles of apoptosis, inflammation, and fibrosis. Hypertension 38: 581-587, 2001.

2. García-Pagán JC, Gracia-Sancho J and Bosch J: Functional aspects on the pathophysiology of portal hypertension in cirrhosis. J Hepatol 57: 458-461, 2012.
3. Kang SH, Kim MY and Baik SK: Novelties in the pathophysiology and management of portal hypertension: New treatments on the horizon. Hepatol Int, Jul 11, 2017 (Epub ahead of print).

4. Kapoor D and Sarin S: Pathophysiology of portal hypertension. J Gastroenterol Hepatol 17 (Suppl): S482-S487, 2002.

5. Halka AT, Turner NJ, Carter A, Ghosh J, Murphy MO, Kirton JP, Kielty CM and Walker MG: The effects of stretch on vascular smooth muscle cell phenotype in vitro. Cardiovasc Pathol 17: 98-102, 2008

6. Kun L, Ying L, Lei W, Jianhua Z, Yongbo X, Tao W, Jinyuan T and Haibo C: Dysregulated apoptosis of the venous wall in chronic venous disease and portal hypertension. Phlebology 31: 729-736, 2016

7. Ma K, Wang H, Yu J, Wei M and Xiao Q: New insights on the regulation of $\mathrm{Ca} 2+$-activated chloride channel TMEM16A. J Cell Physiol 232: 707-716, 2017.

8. Schroeder BC, Cheng T, Jan YN and Jan LY: Expression cloning of TMEM16A as a calcium-activated chloride channel subunit. Cell 134: 1019-1029, 2008.

9. Caputo A, Caci E, Ferrera L, Pedemonte N, Barsanti C, Sondo E, Pfeffer U, Ravazzolo R, Zegarra-Moran O and Galietta LJ: TMEM16A, a membrane protein associated with calcium-dependent chloride channel activity. Science 322: 590-594, 2008

10. Yang YD, Cho H, Koo JY, Tak MH, Cho Y, Shim WS, Park SP, Lee J, Lee B, Kim BM, et al: TMEM16A confers receptor-activated calcium-dependent chloride conductance. Nature 455: 1210-1215, 2008

11. Ruiz C, Martins JR, Rudin F, Schneider S, Dietsche T, Fischer CA Tornillo L, Terracciano LM, Schreiber R, Bubendorf L and Kunzelmann K: Enhanced expression of ANO1 in head and neck squamous cell carcinoma causes cell migration and correlates with poor prognosis. Plos One 7: e43265, 2012.

12. Oh U and Jung J: Cellular functions of TMEM16/anoctamin. Pflugers Arch 468: 443-453, 2016.

13. Forrest AS, Joyce TC, Huebner ML, Ayon RJ, Wiwchar M, Joyce J, Freitas N, Davis AJ, Ye L, Duan DD, et al: Increased TMEM16A-encoded calcium-activated chloride channel activity is associated with pulmonary hypertension. Am J Physiol Cell Physiol 303: C1229-C1243, 2012.

14. Wang $\mathrm{B}, \mathrm{Li} \mathrm{C}$, Huai $\mathrm{R}$ and $\mathrm{Qu} \mathrm{Z}$ : Overexpression of ANO1/TMEM16A, an arterial Ca2+-activated Cl- channel, contributes to spontaneous hypertension. J Mol Cell Cardiol 82: 22-32, 2015.

15. Duvvuri U, Shiwarski DJ, Xiao D, Bertrand C, Huang X, Edinger RS, Rock JR, Harfe BD, Henson BJ, Kunzelmann K, et al: TMEM16A induces MAPK and contributes directly to tumorigenesis and cancer progression. Cancer Res 72: 3270-4281, 2012.

16. Qu Z, Yao W, Yao R, Liu X, Yu K and Hartzell C: The $\mathrm{Ca}(2+)$-activated $\mathrm{Cl}(-)$ channel, ANO1 (TMEM16A), is a double-edged sword in cell proliferation and tumorigenesis. Cancer Med 3: 453-461, 2014.

17. Shiwarski DJ, Shao C, Bill A, Kim J, Xiao D, Bertrand CA, Seethala RS, Sano D, Myers JN, Ha P, et al: To 'grow' or 'go': TMEM16A expression as a switch between tumor growth and metastasis in SCCHN. Clin Cancer Res 20: 4673-4688, 2014.

18. Su EJ, Lombardi DM, Siegal J and Schwartz SM: Angiotensin II induces vascular smooth muscle cell replication independent of blood pressure. Hypertension 31: 1331-1337, 1998.

19. Liu D, Chen J, Wang J, Zhang Z, Ma X, Jia J and Wang Y: Increased expression of urotensin II and GPR14 in patients with cirrhosis and portal hypertension. Int J Mol Med 25: 845-851, 2010.

20. Zimmermann M: Ethical considerations in relation to pain in animal experimentation. Acta Physiol Scand Suppl 554: 221-233, 1986.

21. Xu C and Dong W: Role of hypoxia-inducible factor-1 $\alpha$ in pathogenesis and disease evaluation of ulcerative colitis. Exp Ther Med 11: 1330-1334, 2016.

22. Leik CE, Willey A, Graham MF and Walsh SW: Isolation and culture of arterial smooth muscle cells from human placenta. Hypertension 43: 837-840, 2004.

23. Adhikari N, Shekar KC, Staggs R, Win Z, Steucke K, Lin YW, Wei LN, Alford P and Hall JL; International Society of Cardiovascular Translational Research: Guidelines for the isolation and characterization of murine vascular smooth muscle cells. A report from the international society of cardiovascular translational research. J Cardiovasc Transl Res 8: 158-163, 2015.

24. Zhang L, Liu HJ, Li TJ, Yang Y, Guo XL, Wu MC, Rui YC and Wei LX: Lentiviral vector-mediated siRNA knockdown of SR-PSOX inhibits foam cell formation in vitro. Acta Pharmacol Sin 29: 847-852, 2008 
25. Holt JR, Johns DC, Wang S, Chen ZY, Dunn RJ, Marban E and Corey DP: Functional expression of exogenous proteins in mammalian sensory hair cells infected with adenoviral vectors. J Neurophysiol 81: 1881-1888, 1999.

26. Tacev T, Zaloudik J, Janáková L and Vagunda V: Early changes in flow cytometric DNA profiles induced by californium-252 neutron brachytherapy in squamocellular carcinomas of the uterine cervix. Neoplasma 45: 96-101, 1998.

27. Jin C, Wang A, Chen J, Liu X and Wang G: Relationship between expression and prognostic ability of PTEN, STAT3 and VEGF-C in colorectal cancer. Exp Ther Med 4: 633-639, 2012.

28. Mazzone A, Eisenman ST, Strege PR, Yao Z, Ordog T, Gibbons SJ and Farrugia G: Inhibition of cell proliferation by a selective inhibitor of the $\mathrm{Ca}(2+)$-activated $\mathrm{Cl}(-)$ channel, Ano1 Biochem Biophys Res Commun 427: 248-253, 2012.

29. Hwang SJ, Blair PJ, Britton FC, O'Driscoll KE, Hennig G, Bayguinov YR, Rock JR, Harfe BD, Sanders KM and Ward SM: Expression of anoctamin 1/TMEM16A by interstitial cells of Cajal is fundamental for slow wave activity in gastrointestinal muscles. J Physiol 587: 4887-4904, 2009.

30. Svenningsen $P$, Nielsen MR, Marcussen N, Walter S and Jensen BL: TMEM16A is a $\mathrm{Ca}(2+)$-activated $\mathrm{Cl}(-)$ channel expressed in the renal collecting duct. Acta Physiol (Oxf) 212: 166-174, 2014.

31. Qu Z, Wang B, Zhang Z, Ma L, Li D, Zhuang L, Chi J and Liu J: Functions of ANO1/TMEM16A, Ca2+-activated Cl- channels in regulation of blood pressure and vascular remodeling. J Cardiol Ther 3: 543-548, 2016.

32. Wang M, Yang H, Zheng LY, Zhang Z, Tang YB, Wang GL, Du YH, Lv XF, Liu J, Zhou JG and Guan YY: Downregulation of TMEM16A calcium-activated chloride channel contributes to cerebrovascular remodeling during hypertension by promoting basilar smooth muscle cell proliferation. Circulation 125: 697-707, 2012.

33. Galán M, Varona S, Guadall A, Orriols M, Navas M, Aguiló S, de Diego A, Navarro MA, García-Dorado D, Rodríguez-Sinovas A, et al: Lysyl oxidase overexpression accelerates cardiac remodeling and aggravates angiotensin II-induced hypertrophy. FASEB J 31: 3787-3799, 2017.
34. Li J, Li Y, Zhang Y, Hu D, Gao Y, Shang H and Xing Y: The inhibitory effect of WenxinKeli on H9C2 cardiomyocytes hypertrophy induced by angiotensin II through regulating autophagy activity. Oxid Med Cell Longev 2017: $7042872,2017$.

35. Chen L, Zhao L, Samanta A, Mahmoudi SM, Buehler T, Cantilena A, Vincent RJ, Girgis M, Breeden J, Asante S, et al: STAT3 balances myocyte hypertrophy vis-à-vis autophagy in response to Angiotensin II by modulating the AMPK $\alpha / \mathrm{mTOR}$ axis. PLoS One 12: e0179835, 2017.

36. Lu Y, Guo H, Sun Y, Pan X, Dong J, Gao D, Chen W, Xu Y and $\mathrm{Xu}$ D: Valsartan attenuates pulmonary hypertension via suppression of mitogen activated protein kinase signaling and matrix metalloproteinase expression in rodents. Mol Med Rep 16: 1360-1368, 2017

37. Bataller R, Ginès $P$, Nicolás JM, Görbig MN, Garcia-Ramallo E, Gasull X, Bosch J, Arroyo V and Rodés J: Angiotensin II induces contraction and proliferation of human hepatic stellate cells. Gastroenterology 118: 1149-1156, 2000.

38. Daemen MJ, Lombardi DM, Bosman FT and Schwartz SM: Angiotensin II induces smooth muscle cell proliferation in the normal and injured rat arterial wall. Circ Res 68: 450-456, 1991.

39. Zhang XH, Zheng B, Yang Z, He M, Yue LY, Zhang RN, Zhang M, Zhang W, Zhang X and Wen JK: TMEM16A and myocardin form a positive feedback loop that is disrupted by KLF5 during Ang II-induced vascular remodeling. Hypertension 66: 412-421, 2015.

40. Zhang L, Yang Z, Shi BM, Li DP, Fang CY and Qiu FZ: Expression of local renin and angiotensinogen mRNA in cirrhotic portal hypertensive patient. World J Gastroenterol 9: 1584-1588, 2003.

41. Deng L, Yang J, Chen H, Ma B, Pan K, Su C, Xu F and Zhang J: Knockdown of TMEM16A suppressed MAPK and inhibited cell proliferation and migration in hepatocellular carcinoma. Onco Targets Ther 14: 325-333, 2016.

42. Matrougui K, Eskildsen-Helmond YE, Fiebeler A, Henrion D, Levy BI, Tedgui A and Mulvany MJ: Angiotensin II stimulates extracellular signal-regulated kinase activity in intact pressurized rat mesenteric resistance arteries. Hypertension 36: 617-621, 2000. 